\title{
Recovering piecewise constant refractive indices by a single far-field pattern
}

\section{Blasten, Emilia}

2020-08

Blasten , E \& Liu , H 2020 , ' Recovering piecewise constant refractive indices by a single far-field pattern ' , Inverse Problems , vol. 36 , no. 8 , 085005 . https://doi.org/10.1088/1361-6420/ab958f

http://hdl.handle.net/10138/320394

https://doi.org/10.1088/1361-6420/ab958f

other

acceptedVersion

Downloaded from Helda, University of Helsinki institutional repository.

This is an electronic reprint of the original article.

This reprint may differ from the original in pagination and typographic detail.

Please cite the original version. 


\title{
RECOVERING PIECEWISE CONSTANT REFRACTIVE INDICES BY A SINGLE FAR-FIELD PATTERN
}

\author{
EMILIA BLÅSTEN AND HONGYU LIU
}

\begin{abstract}
We are concerned with the inverse scattering problem of recovering an inhomogeneous medium by the associated acoustic wave measurement. We prove that under certain assumptions, a single farfield pattern determines the values of a perturbation to the refractive index on the corners of its support. These assumptions are satisfied for example in the low acoustic frequency regime. As a consequence if the perturbation is piecewise constant with either a polyhedral nest geometry or a known polyhedral cell geometry, such as a pixel or voxel array, we establish the injectivity of the perturbation to far-field map given a fixed incident wave. This is the first unique determinancy result of its type in the literature, and all of the existing results essentially make use of infinitely many measurements.
\end{abstract}

Keywords: inverse medium scattering; uniqueness; single far-field pattern; value at corner; piecewise constant; polyhedral.

Mathematics Subject Classification (2010): 35P25, 58J50, 35R30, $81 \mathrm{~V} 80$

\section{INTRODUCTION}

1.1. Mathematical setup. Let $V \in L^{\infty}\left(\mathbb{R}^{n}\right), n=2,3$, be a bounded measurable complex-valued function. Let $\Omega$ be a bounded domain in $\mathbb{R}^{n}$ with a connected complement $\mathbb{R}^{n} \backslash \bar{\Omega}$. Assume that $\operatorname{supp}(V) \subset \bar{\Omega}$. Physically speaking, $V$ is the material parameter of an inhomogeneous acoustic medium supported on $\Omega$, with $\Re V$ related to the refractive index and $\Im V$ related to the energy loss into the medium. In what follows, we simply call $V$ the refractive index of the medium. We let $u^{i}$ be an entire solution to the Helmholtz equation,

$$
\left(\Delta+k^{2}\right) u^{i}=0 \text { in } \mathbb{R}^{n} .
$$

Consider the following scattering system for $u \in H_{l o c}^{1}\left(\mathbb{R}^{n}\right)$,

$$
\left\{\begin{array}{l}
\left(\Delta+k^{2}(1+V)\right) u=0 \quad \text { in } \mathbb{R}^{n}, \\
\lim _{r:=|x| \rightarrow+\infty}|x|^{\frac{n-1}{2}}\left(\partial_{r}-i k\right)\left(u-u^{i}\right)=0,
\end{array}\right.
$$

where $\partial_{r}$ is the derivative along the radial direction from the origin. The last limit in 1.2 is known as the Sommerfeld radiation condition and it holds uniformly with respect to the angular variable $\hat{x}:=x /|x| \in \mathbb{S}^{n-1}$ as $r \rightarrow+\infty$. The radiation condition implies the existence of a far-field pattern. More precisely there is a real-analytic function on the unit-sphere at infinity $A_{u^{i}}: \mathbb{S}^{n-1} \rightarrow \mathbb{C}$ such that

$$
u(r \hat{x})=u^{i}(r \hat{x})+\frac{e^{i k r}}{r^{(n-1) / 2}} A_{u^{i}}(\hat{x})+\mathcal{O}\left(\frac{1}{r^{(n+1) / 2}}\right),
$$


which holds uniformly along the angular variable $\hat{x}$.

In the physical scenario, (1.2) describes time-harmonic acoustic scattering due to the presence of an incident wave field $u^{i}$ and an inhomogeneous medium supported on $\Omega$. The functions $u$ and $u^{s}:=u-u^{i}$, respectively, signify the total and scattered wave fields. $A_{u^{i}}$ can be measured by a physical apparatus, and it encodes the information of the scattering medium $V$. An important inverse problem arising in practical applications is to recover $V$ from the knowledge of $A_{u^{i}}(\hat{x})$. This problem lies at the core of many areas of science and technology including radar and sonar, geophysical exploration and medical imaging. In this paper, we are mainly concerned with the unique recovery issue of this inverse problem. That is, given a certain set of measurement data, we shall show what kind of unknowns one can recover; or in other words, given the class of the unknowns, what kind of measurement data can ensure the successful recovery. If one introduces an operator $F$, which sends the unknown $V$ to the associated far-field pattern $A_{u^{i}}$ :

$$
F(V)=A_{u^{i}},
$$

where $F$ is defined by the scattering system 1.2 , then it can readily be shown that $F$ is nonlinear. Hence, the unique recovery result can also ensure the global existence of a unique solution to the nonlinear inverse problem (1.4). In this paper, we are particularly interested in the case with minimal measurement data: a single far-field pattern. By a single far-field pattern, we mean that $A_{u^{i}}(\hat{x})$ is given for all $\hat{x} \in \mathbb{S}^{n-1}$ and a single fixed incident wave $u^{i}$. On the other hand, we note that if $A_{u^{i}}(\hat{x})$ is given for $\hat{x}$ from any open patch of $\mathbb{S}^{n-1}$, then by analytic continuation, it is known for all $\hat{x} \in \mathbb{S}^{n-1}$.

The main result that we aim to establish is that if the medium $V$ is piecewise constant within two general polyhedral geometries, then one can uniquely recover it by a suitable single far-field measurement.

1.2. Connection to existing studies and discussions. For a general medium parameter $V \in L^{\infty}(\Omega)$, the most widely known unique recovery results in the inverse scattering community makes use of infinitely many farfield patterns; see [9, 17] for convenient references. Here, by infinitely many far-field patterns, we mean $A_{u^{i}}$ are given corresponding to incident plane waves $u^{i}=e^{i k x \cdot d}$ with all possible $d \in \mathbb{S}^{n-1}$ and a fixed $k \in \mathbb{R}_{+}$. A crucial ingredient is that the data set $\left\{A_{e^{i k x \cdot d}}(\hat{x})\right\}_{(\hat{x}, d) \in \mathbb{S}^{n-1} \times \mathbb{S}^{n-1}}$ is equivalent to the Cauchy data set, $\mathcal{C}_{V}:=\left\{\left(\left.u\right|_{\partial \Omega},\left.\partial_{\nu} u\right|_{\partial \Omega}\right) ;\left(\Delta+k^{2}(1+V)\right) u=0\right.$ in $\left.\Omega\right\}$, where $\nu \in \mathbb{S}^{n-1}$ is the exterior unit normal vector to $\partial \Omega$, cf. 21.28. Hence, in the case with infinitely many measurement, the study of the inverse scattering problem of recovering $V$ can be reduced to the study of the inverse boundary value problem of recovering $V$ from the associated Cauchy data set. The establishment of the unique recovery result for the aforementioned inverse boundary value problem is mainly based on the Sylvester-Uhlmann method pioneered in [25], and on the Bukhgeim method in two dimensions [8]. We also refer to a recent survey paper [27] for many subsequent relevant developments.

The problem of recovering a potential from the Cauchy data is formally overdetermined in three and higher dimensions. This manifests in a much 
tardier solution to the problem in the plane 8 than in higher dimensions 25]. In a similar vein, the conjectured integrability limit of $L^{n / 2}$ for potential recovery [18] has been achieved in the formally overdetermined case [12], but the two dimensional formally determined case is lagging behind $[2,6$. Also, the formally determined backscattering problem is still largely open, but with recent progress on an admissible class of potentials [23,24. In two recent articles by one of the authors [14, 20, it is shown that if the medium $V$ is from a certain special class and contains an impenetrable obstacle, then $\left\{A_{e^{i k x \cdot d}}(\hat{x})\right\}_{(\hat{x}, k) \in \mathbb{S}^{n-1} \times \mathbb{R}_{+}}$with a fixed $d \in \mathbb{S}^{n-1}$ and $k$ in an interval is enough measurement data. This problem is formally determined. The problem of our paper - the unique determination of a potential by a single far-field measurement - is formally underdetermined, so stands no chance of being completely solved. However by restricting the potentials into a space that's still useful with respect to applications, but has fewer degrees of freedom, this problem is avoided.

Making use of the corner scattering results in 3, 4, 7, 13], the authors in 5, 15 showed that if a medium $V$ is supported on a convex polyhedral domain $\Omega$, then $\Omega$ can be uniquely determined (in a stable way) by a single far-field pattern. Earlier work on the scattering support also recover useful information from a single far-field pattern [19]. In this article we show that not only the domain, but also the values of the potential can be uniquely determined at the vertices by a far-field pattern created by a suitable incident wave. As a consequence, we are able to establish a much more general result in the same class of measurements: determining a polyhedral piecewise-constant medium parameter by a single far-field pattern.

If the medium is piecewise constant on a suitable known polyhedral grid, for example a pixel or voxel array, then one can uniquely recover it by a single far-field pattern. If the grid is not known a-priori, then we can recover the potential assuming that it is piecewise constant on a nested polyhedral grid. To the best of our knowledge, this is the first uniqueness result of its type in the literature.

There is one important implication of our uniqueness result, particularly from the numerical point of view. The finite element method (FEM) is widely used in the engineering community. Using the FEM approximation, the medium parameter $V$ is usually approximated by a piecewise polynomial function with a polyhedral triangulation of its support. That is,

$$
V \approx V_{h}:=\sum_{\Sigma_{j} \in \mathcal{T}_{h}} P_{j} \chi_{\Sigma_{j}}
$$

where $\mathcal{T}_{h}$ is a polyhedral triangulation of $\Omega$ and $P_{j}$ is a polynomial function supported in $\Sigma_{j}$, a.k.a a finite element basis function. Here, $h \in \mathbb{R}_{+}$signifies the mesh size of the FEM approximation. Hence, under the approximation (1.5), instead of solving (1.4), one actually solves

$$
F\left(V_{h}\right)=A_{u^{i}}\left(V_{h}\right)+\epsilon_{h}, \quad \epsilon_{h}:=A_{u^{i}}(V)-A_{u^{i}}\left(V_{h}\right) .
$$

Clearly, by using our uniqueness result, if the approximation is made with a piecewise constant function associated to a certain polyhedral triangulation as discussed in this work, there is a one-to-one correspondence between 
$V_{h}$ and $A_{u^{i}}\left(V_{h}\right)$ through the far-field map $F$, which shall be of critical importance in analyzing the convergence of the FEM approximation (1.6) . Hence, the theoretical results obtained in the current article might motivate some novel numerical and practical applications, which are definitely worth further investigation.

We are also led to the following conjecture in inverse scattering theory: with one or a few incident plane waves, if two mediums $V$ and $V^{\prime}$ of certain type are different, then the associated far-field patterns should be distinguishable from each other. At least, according to the current article, the conjecture is true for piecewise constant mediums supported in certain polyhedral domains. However it is known to be false for some radially symmetric mediums 10,11 . We believe that the conjecture would hold for a general class of mediums, say, with piecewise polynomial material parameters. We shall investigate this and other interesting issues in our future work.

1.3. Improvements to the method. Finally, we would like to comment on the mathematical argument in deriving our uniqueness results. In addition to the completely new potential determination theorems, we improve past corner scattering methods. The first step is to show that not only can the vertices of the scatterer be recovered, but also the value of the potential on these points. This is an extremely useful improvement over past determination results [5, 13, 15]. For this we will have to use the total wave of one of the potentials as an incident wave for the other one. Technically we have to use the Taylor expansion of such a non-smooth wave. This difficulty is avoided by making sure that the total wave does not vanish at vertices.

A significant technical improvement to the corner scattering method is the following: our results will now work for arbitrary convex polyhedrons in 3D, hence improving the recovery results of [5, 15] where the three dimensional objects had to be rectangular boxes. This generalization is made possible by studying more carefully the Laplace transforms of polyhedral cones, as inspired by [1], and works when the total wave is nonzero at the corner. See Lemma 3.5. We leave for future work the technically much more challenging problem of dealing with total waves that vanish to arbitrarily high order at some of the vertices of the scatterers.

Once the corners and values at the corners are recovered, this means that we know the piecewise constant potential in a neighbourhood of its corners. To progress further we need to propagate the equality of the total waves of the two potentials. This is done by an application of Holmgren's uniqueness theorem. As an aside, what we prove implies a variation of Holmgren's theorem in the setting of two equations, $(\Delta+q) u=0$ and $\left(\Delta+q^{\prime}\right) u^{\prime}=0$ : if we know that $u=u^{\prime}$ and $\partial_{\nu} u=\partial_{\nu} u^{\prime}$ on the boundary near a corner, then under the assumptions of our theorems we have $u=u$ inside, and also $q=q^{\prime}$ at the corner.

We state three theorems in this paper. One is the unique recovery of shape and values at the vertices of a polyhedral scatterer. The second one is the complete recovery of a piecewise constant potential defined on an a-priori known grid of polyhedral cells. Think of pixels for example. The last theorem shows a case where even the cell structure is not known beforehand, however we must have the knowledge that the potentials have a nested geometry. 
These are not the ultimate limits of this method, however it is still unclear how far can one go.

The rest of the paper is organized as follows. In Section 2, we present the main uniqueness results. Section 3 is devoted to the proofs.

\section{MAin RESUlts}

2.1. Geometric setup. We shall consider the inverse problem (1.4) with a piecewise constant refractive index of the following form

$$
V=\sum_{j} V_{j} \chi_{\Sigma_{j}}, \quad \bar{\Omega}=\bigcup_{j} \overline{\Sigma_{j}},
$$

where $V_{j} \in \mathbb{C}$ are constants, and $\Sigma_{j}$ are mutually disjoint bounded open subsets in $\mathbb{R}^{n}$. For completeness we also recover the corner value of potentials that are Hölder-continuous near the vertices on a polygonal domain.

In the piecewise constant case, we shall consider two geometric setups of the subdomains $\Sigma_{j}$ that can be described as follows. The first one is referred to as the polyhedral cell geometry, see Figure 1.(A) for a schematic illustration.

Definition 2.1. An admissible cell $P \subset \mathbb{R}^{n}$ is a bounded open convex polytope, i.e. a polygon in $2 \mathrm{D}$ and a polyhedron in $3 \mathrm{D}$.

Definition 2.2. For $j \in \mathbb{Z}_{+}$let each of $\Sigma_{j} \subset \mathbb{R}^{n}$ be an admissible cell or the empty set, $\cup_{j} \overline{\Sigma_{j}}$ is simply connected, bounded and $\Sigma_{j} \cap \Sigma_{k}=\emptyset$ if $j \neq k$. A bounded potential $V \in L^{\infty}\left(\mathbb{R}^{n}\right)$ is said to be piecewise constant with polyhedral cell geometry if

(1) there are constants $V_{j} \in \mathbb{C}$ such that

$$
V(x)=\sum_{j=1}^{\infty} V_{j} \chi_{\Sigma_{j}}(x),
$$

with $V_{j}=0$ if $\Sigma_{j}=\emptyset$, and

(2) each $\Sigma_{j} \neq \emptyset$ has a vertex that can be connected to infinity by a path that stays distance $d \geq d_{0}>0$ from any $\Sigma_{k}$ with $k>j$.

The latter condition is satisfied for example by taking any finite number of unit squares or cubes that are part of a lattice, and ordering them from left to right and top to bottom. In essence if we approximate a potential's graph by a discrete picture consisting of pixels or voxels, then this approximation represents a piecewise constant potential with polyhedral cell geometry.

The second one is referred to as the polyhedral nested geometry. See Figure 1.(B) for a schematic illustration.

Definition 2.3. For $j \in \mathbb{Z}_{+}$let each of $D_{j} \subset \mathbb{R}^{n}$ be an admissible cell or the empty set, and

$$
D_{j} \ni D_{j+1} .
$$

A bounded potential $V \in L^{\infty}\left(\mathbb{R}^{n}\right)$ is said to be piecewise constant with polyhedral nested geometry if there are constants $V_{j} \in \mathbb{C}$, with $V_{1} \neq 0$, $V_{j+1} \neq V_{j}$ such that

$$
V(x)=\sum_{j=1}^{\infty} V_{j} \chi_{\Sigma_{j}}(x)
$$




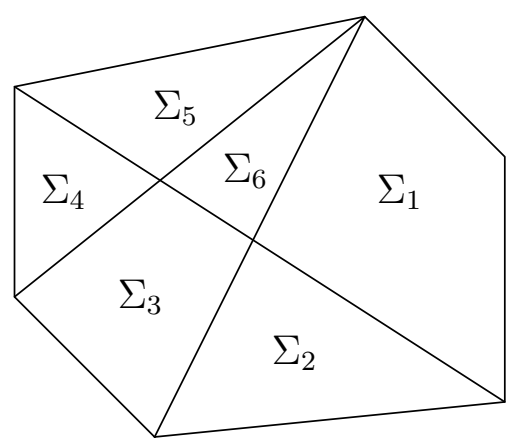

(A)

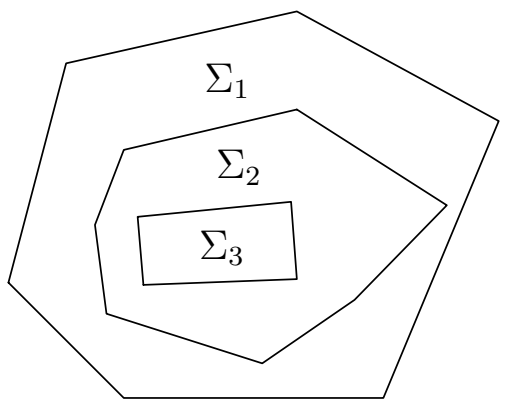

(B)

Figure 1. Schematic illustration of the two polyhedral geometries in two dimensions. (A) Polyhedral cell geometry; (B) Polyhedral nested geometry.

where $\Sigma_{j}=D_{j} \backslash \overline{D_{j+1}}$.

Finally we define potentials analogous to ones used in previous corner scattering results $[15,22]$.

Definition 2.4. A potential $V \in L^{\infty}$ is a non-constant admissible potential if there is an admissible cell $P \subset \mathbb{R}^{n}$ and bounded function $\varphi \in L^{\infty}\left(\mathbb{R}^{n}\right)$ such that $V=\chi_{P} \varphi$. Moreover we require that $\varphi$ be Hölder $C^{\alpha}$-continuous in a neighbourhood of each of the vertices of $P$ with $\alpha>0$ in $2 \mathrm{D}$ and $\alpha>1 / 4$ in 3D. Finally, the function $\varphi$ must not vanish at any of the vertices.

2.2. Unique recovery results. Our results depend on the total field $u$ not vanishing at the vertices of the various polytopes $\Sigma_{j}$. The nodal (or vanishing) set cannot be too large in general. For concreteness Lemma 3.2 gives a sufficient condition: the nodal set is empty for low enough frequencies with incident plane-waves; see Remark 3.3 for more relevant discussion.

Theorem 2.5. Let $n \in\{2,3\}, k>0$ and $V=\chi_{P} \varphi, V^{\prime}=\chi_{P^{\prime}} \varphi^{\prime}$ be two non-constant admissible potentials.

Let $u^{i}$ be an incident wave such that $u\left(x_{c}\right) \neq 0$ or $u^{\prime}\left(x_{c}\right) \neq 0$ for the total waves $u, u^{\prime}$ at each vertex $x_{c}$ of $P$ or $P^{\prime}$. Assume that

$$
A_{u^{i}}=A_{u^{i}}^{\prime}
$$

for the far-field patterns arising from $V$ and $V^{\prime}$, respectively. Then $P=P^{\prime}$ and $\varphi\left(x_{c}\right)=\varphi^{\prime}\left(x_{c}\right)$ on each vertex $x_{c}$ of $P=P^{\prime}$.

Theorem 2.6. Let $n \in\{2,3\}$ and $k>0$. Let $V$ and $V^{\prime}$ be two piecewise constant potentials with common polyhedral cell geometry.

Let $u^{i}$ be an incident wave such that $u\left(x_{c}\right) \neq 0$ or $u^{\prime}\left(x_{c}\right) \neq 0$ for each vertex $x_{c}$ of the cells of $V$ and $V^{\prime}$. If $A_{u^{i}}=A_{u^{i}}^{\prime}$ then $V=V^{\prime}$.

Theorem 2.7. Let $n \in\{2,3\}$ and $k>0$. Let $V$ and $V^{\prime}$ be two piecewise constant potentials with polyhedral nested geometry.

Let $u^{i}$ be an incident wave such that $u\left(x_{c}\right) \neq 0$ or $u^{\prime}\left(x_{c}\right) \neq 0$ for each vertex $x_{c}$ of the cells of $V$ and $V^{\prime}$. If $A_{u^{i}}=A_{u^{i}}^{\prime}$ then $V=V^{\prime}$. 


\section{Proofs}

We first present a useful lemma.

Lemma 3.1. Let $u \in H_{\text {loc }}^{1}\left(\mathbb{R}^{n}\right)$ be the solution to 1.2 . Suppose that $\Omega \subset$ $B_{R}$, where $B_{R}$ is a central ball of radius $R \in \mathbb{R}_{+}$. Then $u^{s} \in H^{2}\left(B_{R}\right)$ and there exists $C_{0} \in \mathbb{R}_{+}$such that when $k^{2}\|V\|_{\infty} \leq C_{0}$,

$$
\left\|u^{s}\right\|_{H^{2}\left(B_{R}\right)} \leq C k^{2}\|V\|_{L^{\infty}(\Omega)}\left\|u^{i}\right\|_{L^{2}(\Omega)}
$$

where $C$ is a positive constant depending only on $R$.

Proof. We only prove the case with $n=3$, and the other cases can be proved by following a similar argument. Let

and define

$$
\Phi(x)=\frac{1}{4 \pi} \frac{e^{i k|x|}}{|x|}, \quad x \in \mathbb{R}^{3} \quad|x| \neq 0 .
$$

$$
\mathcal{L}_{V}(u)=\int_{\Omega} \Phi(x-y) V(y) u(y) d y .
$$

We know there is the following integral relation (cf. [9])

$$
u^{s}(x)=k^{2} \mathcal{L}_{V}\left(u^{s}\right)(x)+k^{2} \mathcal{L}_{V}\left(u^{i}\right)(x), \quad x \in \mathbb{R}^{3} .
$$

Using the facts that $u^{s} \in H^{1}\left(B_{R}\right)$, and $\mathcal{L}_{V}$ maps $L^{2}\left(B_{R}\right)$ continuously into $H^{2}\left(B_{R}\right)$ with norm $k^{2}\|V\|_{\infty}$ (cf. Theorem 8.2 in 9$]$ ), one can easily see that $u^{s} \in H^{2}\left(B_{R}\right)$. For $k$ or $\|V\|_{\infty}$ sufficiently small, we have from 3.2 that $u^{s}=\left(I-k^{2} \mathcal{L}_{V}\right)^{-1}\left(k^{2} \mathcal{L}_{V}\left(u^{i}\right)\right)$ and hence

$$
\left\|u^{s}\right\|_{L^{2}\left(B_{R}\right)} \leq C k^{2}\|V\|_{L^{\infty}(\Omega)}\left\|u^{i}\right\|_{L^{2}(\Omega)},
$$

where $C$ is positive constant depending only on $R$. Finally, by applying the estimate in (3.3) to the RHS of (3.2), together with the use of the mapping property of $\mathcal{L}_{V}$ again, one can show (3.1). The proof is complete.

Lemma 3.2. Let $n \in\{2,3\}, k>0$ and let $B_{R}$ be a central ball of radius $R \in \mathbb{R}_{+}$. There is $C>0$ such that if $V \in L^{\infty}\left(B_{R}\right)$ with

$$
k^{2}\|V\|_{\infty}<C
$$

and $u \in H^{2}\left(B_{R}\right)$ is the total field created by $V$ and an incident unit-modulus plane-wave $u^{i}$ of wave-number $k$, then $u$ cannot vanish in $B_{R}$.

Proof. Lemma 3.1 and the Sobolev embedding of $H^{2}$ into $L^{\infty}$ in 2D and 3D imply that $\left\|u^{s}\right\|_{\infty}<1$ in $B_{R}$ then. Hence

$$
|u(x)| \geq\left|u^{i}(x)\right|-\left|u^{s}(x)\right|>0 .
$$

for any $x \in B_{R}$.

Remark 3.3. It is remarked that Lemma 3.2 provides a sufficient scenario for the non-vanishing of the total wave fields at the vertex points, which is required in Theorems 2.5 and 2.6. According to (3.4), if the wave-number is sufficiently small compared to the refractive index, then the non-vanishing requirement is fulfilled. This is the only place where we make use of the low wave-number assumption. In our subsequent analysis, say Lemmas 3.5 and 3.6, the CGO (complex geometric optics) argument does not depend on the wave-number $k$ as long as it is fixed. Moreover, we would like to point out 
that the non-vanishing of the total wave fields may hold in more general scenarios without this low wave-number assumption.

The following lemma is a key identity in corner scattering. It is a slight modification of the Alessandrini identity that is used in inverse problems in general. It becomes very useful when $V$ and $V^{\prime}$ have their supports on a common cone, and $u=u^{\prime}$ outside that cone. Letting $u_{0}$ be a complex geometrical optics (CGO) solution is a good choice then.

Lemma 3.4. Let $U \subset \mathbb{R}^{n}$ be a bounded Lipschitz domain and $q, q^{\prime} \in L^{\infty}(U)$. If $u_{0}, u, u^{\prime} \in H^{2}(U)$ satisfy

$$
\begin{aligned}
(\Delta+q) u_{0} & =0 \\
(\Delta+q) u & =0 \\
\left(\Delta+q^{\prime}\right) u^{\prime} & =0
\end{aligned}
$$

in $U$ then

$$
\int_{U}\left(q-q^{\prime}\right) u^{\prime} u_{0} d x=\int_{\partial U}\left(u_{0} \partial_{\nu}\left(u-u^{\prime}\right)-\left(u-u^{\prime}\right) \partial_{\nu} u_{0}\right) d \sigma .
$$

Proof. We have $(\Delta+q)\left(u^{\prime}-u\right)=\left(q-q^{\prime}\right) u^{\prime}$. By Green's identities

$$
\begin{aligned}
\int_{U}\left(q-q^{\prime}\right) u^{\prime} u_{0} d x=\int_{U} u_{0}(\Delta+q)\left(u^{\prime}-u\right) d x \\
\quad=\int_{\partial U}\left(u_{0} \partial_{\nu}\left(u-u^{\prime}\right)-\left(u-u^{\prime}\right) \partial_{\nu} u_{0}\right) d \sigma+\int_{U}\left(u^{\prime}-u\right)(\Delta+q) u_{0} d x
\end{aligned}
$$

and the claim follows because $(\Delta+q) u_{0}=0$.

Another key-element in the proofs of corner scattering is to show that the Laplace transforms of the indicator functions of certain cones do not vanish when evaluated at the complex geometrical optics parameter $\rho \in \mathbb{C}^{n}$, $\rho \cdot \rho=0$. Here we generalize previous results of [5] to allow for arbitrary convex polyhedrons in 3D. All previous papers on the topic, excluding [13] had required that the three dimensional polyhedra were rectangular boxes. The following technique of splitting the Laplace transform into a simple but large integral, and a difficult but bounded one was inspired by [1].

Lemma 3.5. Let $n \in\{2,3\}$ and $\mathcal{C} \subset \mathbb{R}^{n}$ be an open convex polyhedral cone smaller than a half-space. Then there is $\rho \in \mathbb{C}^{n}, \rho \cdot \rho=0, \rho \neq 0$ such that

$$
\int_{\mathcal{C}} e^{\rho \cdot x} d x \neq 0
$$

and the integral converges.

Proof. We may assume that the vertex of $\mathcal{C}$ is the origin. If $\mathcal{C}$ is a simplex cone, i.e. it is generated by $n$ linearly independent unit vectors

$$
w_{1}, \ldots, w_{n} \in \mathbb{S}^{n-1}, \quad \mathcal{C}=\left\{\alpha_{1} w_{1}+\ldots+\alpha_{n} w_{n} \mid \alpha_{j}>0\right\},
$$

then by a linear change of variables we see that

$$
\int_{\mathcal{C}} e^{\rho \cdot x} d x=\frac{\left|w_{1} \wedge \ldots \wedge w_{n}\right|}{\left(-\rho \cdot w_{1}\right) \cdot \ldots \cdot\left(-\rho \cdot w_{n}\right)}
$$


where $\left|w_{1} \wedge \ldots \wedge w_{n}\right|$ is the determinant of the $n \times n$ matrix with column vectors $w_{1}, \ldots, w_{n}$. The two dimensional claim follows immediately by letting $-\Re \rho$ be in the interior of $\mathcal{C}$.

For the three dimensional case let the generators of $\mathcal{C}$ be

$$
w_{1}, \ldots, w_{m}
$$

in other words $\left\{t w_{j} \mid t>0\right\}$ are its edges. We may order these vectors so that the segments $\left\{t w_{j}+(1-t) w_{j+1}\right\}$ and $\left\{t w_{m}+(1-t) w_{1}\right\}$ are on $\partial \mathcal{C}$. Fix $w=w_{1}$ and let $z$ be a unit vector such that

$$
z \cdot w=0, \quad z \cdot w_{j}>0
$$

for $j>1$. For $\varepsilon>0$ let

$$
R_{\varepsilon}=\frac{z+\varepsilon w}{|z+\varepsilon w|}
$$

and let $I$ be a unit vector such that $I \cdot z=I \cdot w=0$. Finally let

$$
\rho=-R_{\varepsilon}-i I
$$

and we observe that $\rho \cdot \rho=0, \rho \neq 0$. Moreover we note that the Laplace transform converges when $\varepsilon$ is small enough but positive.

Let $\mathcal{C}_{1}$ be the open cone generated by $w=w_{1}, w_{2}$ and $w_{m}$. We have

$$
\begin{aligned}
& \rho \cdot w_{1}=\frac{-\varepsilon}{|z+\varepsilon w|} \longrightarrow 0 \\
& \rho \cdot w_{j}=-\frac{z \cdot w_{j}+\varepsilon w \cdot w_{j}}{|z+\varepsilon w|}-i I \cdot w_{j} \longrightarrow-z \cdot w_{j}-i I \cdot w_{j}
\end{aligned}
$$

for $j \in\{2, m\}$ as $\varepsilon \rightarrow 0$. Hence $\left|\rho \cdot w_{j}\right| \geq\left|z \cdot w_{j}\right|>0$. Thus by the formula for the transform of a simplex cone

$$
\lim _{\varepsilon \rightarrow 0}\left|\int_{\mathcal{C}_{1}} e^{\rho \cdot x} d x\right|=\infty .
$$

We shall prove that the integral over $\mathcal{C}^{\prime}=\mathcal{C} \backslash \overline{\mathcal{C}_{1}}$ is bounded next. This cone is generated by the vectors $w_{2}, \ldots, w_{m}$. There is a positive constant $\delta\left(w_{2}, \ldots, w_{m}\right)$ such that if $\theta \in \mathcal{C}^{\prime}$ is a unit vector then

$$
\theta=\alpha_{2} w_{2}+\ldots+\alpha_{m} w_{m}, \quad \max _{j} \alpha_{j} \geq \delta\left(w_{2}, \ldots, w_{m}\right)>0
$$

for some non-negative real numbers $\alpha_{j}$. Then

$$
\begin{gathered}
\lim _{\varepsilon \rightarrow 0} \Re \rho \cdot \theta=-\sum_{j=2}^{m} \alpha_{j} z \cdot w_{j} \leq-\min _{j \geq 2} z \cdot w_{j} \sum_{j=2}^{m} \alpha_{j} \\
\leq-\min _{j \geq 2} z \cdot w_{j} \delta\left(w_{2}, \ldots, w_{m}\right)<0
\end{gathered}
$$

by the choice of $z$. By the triangle inequality

$$
\left|\int_{\mathcal{C}^{\prime}} e^{\rho \cdot x} d x\right| \leq \int_{\mathcal{C}^{\prime} \cap \mathbb{S}^{2}} \int_{0}^{\infty} e^{\Re \rho \cdot \theta r} r^{2} d r d \sigma(\theta)
$$

and hence, writing $\delta\left(z, w_{2}, \ldots, w_{m}\right)=\min _{j \geq 2} z \cdot w_{j} \delta\left(w_{2}, \ldots, w_{m}\right)$, we get

$$
\lim _{\varepsilon \rightarrow 0}\left|\int_{\mathcal{C}^{\prime}} e^{\rho \cdot x} d x\right| \leq \sigma\left(\mathcal{C}^{\prime} \cap \mathbb{S}^{2}\right) \int_{0}^{\infty} e^{-\delta\left(z, w_{2}, \ldots, w_{m}\right) r} r^{2} d r=C\left(z, w_{2}, \ldots, w_{m}\right)
$$

the latter of which is a finite constant. 
The integral over $\mathcal{C}_{1}$ can be made arbitrarily large while the integral over $\mathcal{C} \backslash \overline{\mathcal{C}_{1}}$ stays uniformly bounded. Hence the whole integral can be made arbitrarily large, and thus nonzero.

Lemma 3.6. Let $\Omega \subset \mathbb{R}^{n}, n \in\{2,3\}$ be a bounded domain. Let $q, q^{\prime} \in$ $L^{\infty}(\Omega)$ and let $u, u^{\prime} \in H^{2}(\Omega)$ solve

$$
(\Delta+q) u=0, \quad\left(\Delta+q^{\prime}\right) u^{\prime}=0
$$

in $\Omega$. Let $x_{c} \in \partial \Omega$ be a point for which there is a neighbourhood $B$ and an admissible cell $\Sigma$ having $x_{c}$ as vertex such that $B \cap \Omega=B \cap \Sigma$.

Assume that $u=u^{\prime}$ and $\partial_{\nu} u=\partial_{\nu} u^{\prime}$ on $B \cap \partial \Omega$. If $q$ and $q^{\prime}$ are $C^{\alpha}$ uniformly Hölder-continuous functions with $\alpha>0$ in $2 D$ and $\alpha>1 / 4$ in $3 D$ in $B \cap \Omega$ then

$$
\left(q-q^{\prime}\right)\left(x_{c}\right) u\left(x_{c}\right)=\left(q-q^{\prime}\right)\left(x_{c}\right) u^{\prime}\left(x_{c}\right)=0 .
$$

Proof. Choose coordinates such that $x_{c}=\overline{0}$. We may assume that $B$ is a small $x_{c}$-centred disc with $B \cap \Omega=B \cap \mathcal{C}$ for some open cone $\mathcal{C} \subset \mathbb{R}^{n}$ with vertex $x_{c}$. We shall prove the case $\left(q-q^{\prime}\right)\left(x_{c}\right) u^{\prime}\left(x_{c}\right)=0$. The other one follows by symmetry.

For any $\rho \in \mathbb{C}^{n}$ with $\rho \cdot \rho=0$ and $|\Re \rho|>0$ large enough depending on $q, B, \mathcal{C}$, the equation

$$
(\Delta+q) u_{0}=0
$$

has a complex geometrical optics solution $u_{0}(x)=\exp (\rho \cdot x)(1+\psi(x))$ in $B \cap \mathcal{C}$. This is given for example by Theorem 3.1 in 22 for $2 \mathrm{D}$, Proposition 7.6 in [5] or Lemmas 2.1 and 3.2 in [15], all of them requiring $\alpha>1 / 4$ in 3D. They imply the existence of $p \geq 2$ and $\delta>0$, both independent of $\rho$, such that

and $u_{0} \in H^{2}(B \cap \mathcal{C})$.

$$
\|\psi\|_{L^{p}(B \cap \mathcal{C})} \leq C|\Re \rho|^{-n / p-\delta}
$$

Recall that $u=u^{\prime}$ and $\partial_{\nu} u=\partial_{\nu} u^{\prime}$ on $B \cap \partial \mathcal{C}$. Lemma 3.4 gives

$$
\int_{B \cap \mathcal{C}}\left(q-q^{\prime}\right) u^{\prime} u_{0} d x=\int_{\mathcal{C} \cap \partial B}\left(u_{0} \partial_{\nu}\left(u-u^{\prime}\right)-\left(u-u^{\prime}\right) \partial_{\nu} u_{0}\right) d \sigma
$$

Choose $\rho$ such that $\exp (\Re \rho \cdot x)$ decays exponentially in the cone $\mathcal{C}$. Then the boundary integral's absolute value can be estimated as

$$
\begin{aligned}
|\ldots| & \leq\left\|u-u^{\prime}\right\|_{H^{2}(B \cap \mathcal{C})}\left\|u_{0}\right\|_{H^{2}(B \cap \mathcal{C})} \\
& \leq C\left\|u-u^{\prime}\right\|_{H^{2}(B \cap \mathcal{C})}(1+|\Re \rho|) e^{-c|\Re \rho|}\left(1+\|\psi\|_{H^{2}(B \cap \mathcal{C})}\right) \\
& \leq C e^{-c^{\prime}|\Re \rho|}
\end{aligned}
$$

when $|\Re \rho|$ is large enough: $\|\psi\|_{H^{2}}$ is of the order $|\Re \rho|^{2}$ and by Lemma 3.1 the $H^{2}$ norm of $u-u^{\prime}=u^{s}-u^{\prime s}$ is finite. Let us split and estimate the integral over $B \cap \mathcal{C}$ next. Write $\delta_{q}=q-q^{\prime}$ and split

$$
\begin{aligned}
\left(q-q^{\prime}\right)(x) & =\delta_{q}(\overline{0})+\left(\delta_{q}(x)-\delta_{q}(\overline{0})\right), \\
u^{\prime}(x) & =u^{\prime}(\overline{0})+\left(u^{\prime}(x)-u^{\prime}(\overline{0})\right), \\
u_{0}(x) & =e^{\rho \cdot x}+e^{\rho \cdot x} \psi(x),
\end{aligned}
$$


where the pointwise values of $u^{\prime}$ are well-defined since $H^{2}$-functions are continuous in $2 \mathrm{D}$ and $3 \mathrm{D}$. We have the estimates

$$
\begin{aligned}
\left|\delta_{q}(x)-\delta_{q}(\overline{0})\right| & \leq\left(\|q\|_{C^{\alpha}}+\left\|q^{\prime}\right\|_{C^{\alpha}}\right)|x|^{\alpha}, \\
\left|u^{\prime}(x)-u^{\prime}(\overline{0})\right| & \leq C_{B}\left\|u^{\prime}\right\|_{H^{2}(B)}|x|^{1 / 2}, \\
\|\psi\|_{L^{p}(B)} & \leq C|\Re \rho|^{-n / p-\delta}
\end{aligned}
$$

by the definition of Hölder-continuity, the Sobolev embedding of $H^{2}(B)$ into the space $C^{1 / 2}$ of Hölder-continuous functions in $2 \mathrm{D}$ and $3 \mathrm{D}$, and the previous paragraph about the complex geometrical optics solution.

If $\rho$ is such that the integral $\int_{\mathcal{C}} \exp (\rho \cdot x) d x$ converges, we see the following telescope identity:

$$
\begin{aligned}
\left(q-q^{\prime}\right)(\overline{0}) u^{\prime}(\overline{0}) \int_{\mathcal{C}} e^{\rho \cdot x} d x=\left(q-q^{\prime}\right)(\overline{0}) u^{\prime}(\overline{0}) \int_{\mathcal{C} \backslash B} e^{\rho \cdot x} d x \\
\quad-u^{\prime}(\overline{0}) \int_{B \cap \mathcal{C}} e^{\rho \cdot x}\left(\delta_{q}(x)-\delta_{q}(\overline{0})\right) d x \\
\quad-\int_{B \cap \mathcal{C}} e^{\rho \cdot x}\left(q-q^{\prime}\right)(x)\left(u^{\prime}(x)-u^{\prime}(\overline{0})\right) d x \\
\quad-\int_{B \cap \mathcal{C}} e^{\rho \cdot x}\left(q-q^{\prime}\right)(x) u^{\prime}(x) \psi(x) d x \\
+\int_{\mathcal{C} \cap \partial B}\left(u_{0} \partial_{\nu}\left(u-u^{\prime}\right)-\left(u-u^{\prime}\right) \partial_{\nu} u_{0}\right) d \sigma .
\end{aligned}
$$

We will estimate the various terms in the identity above next. The first and last term decay exponentially as $|\Re \rho| \rightarrow \infty$ since $\Re \rho \cdot x \leq-c|\Re \rho||x|$ for $x \in \mathcal{C}$ is satisfied when $\int_{\mathcal{C}} \exp (\rho \cdot x) d x$ is finite. For the three other terms note that

$$
\left.\left.\left|\int_{\mathcal{C}} e^{\rho \cdot x}\right| x\right|^{s} d x\left|\leq C \int_{0}^{\infty} e^{-c|\Re \rho| r} r^{s+n-1} d r=\right| \Re \rho\right|^{-n-s} \int_{0}^{\infty} e^{-c r^{\prime}} r^{\prime n+s-1} d r^{\prime}
$$

and similarly

$$
\left\|e^{\rho \cdot x}\right\|_{L^{p^{\prime}}(\mathcal{C})} \leq\left(\int_{\mathcal{C}} e^{-c|\Re \rho||x| p^{\prime}} d x\right)^{1 / p^{\prime}}=C|\Re \rho|^{-n / p^{\prime}} .
$$

Using the triangle and Hölder's inequalities with exponent $\left(p, p^{\prime}\right)$ where $1 / p+$ $1 / p^{\prime}=1$ and the estimates for $\delta_{q}$ and $u^{\prime}$ from the previous paragraph, we see that

$$
\begin{aligned}
& \left|\left(q-q^{\prime}\right)(\overline{0}) u^{\prime}(\overline{0}) \int_{\mathcal{C}} e^{\rho \cdot x} d x\right| \\
& \quad \leq C\left(e^{-c|\Re \rho|}+|\Re \rho|^{-n-\alpha}+|\Re \rho|^{-n-1 / 2}+|\Re \rho|^{-n-\delta}\right)
\end{aligned}
$$

for $|\Re \rho|$ large engouh.

For the lower bound note that no matter how large we require $|\Re \rho|$ to be, Lemma 3.5 and a simple multiplication by a real number give the existence of $\rho$ satisfying all the requirements stated previously, and that

$$
\left|\int_{\mathcal{C}} e^{\rho \cdot x} d x\right| \geq C|\Re \rho|^{-n}
$$

for some $C \neq 0$. This implies that $\left(q-q^{\prime}\right)(\overline{0}) u^{\prime}(\overline{0})=0$. 
Proposition 3.7. Let $D \subset \mathbb{R}^{n}, n \in\{2,3\}$, be a bounded domain and $P, P^{\prime} \Subset D$ admissible cells. Let $\alpha>0$ in $2 D$ and $\alpha>1 / 4$ in $3 D$. Let $q, q^{\prime} \in L^{\infty}(D)$ be uniformly $C^{\alpha}$ Hölder continuous outside $P, P^{\prime}$, respectively and $q=q^{\prime}$ outside $P \cup P^{\prime}$. Assume that $q$ restricted to $P$ is uniformly $C^{\alpha}$ Hölder continuous near each vertex $x_{c}$ of $P$, but that

$$
\lim _{\substack{x \in P \\ x \rightarrow x_{c}}} q(x) \neq \lim _{\substack{x \in D \backslash \bar{P} \\ x \rightarrow x_{c}}} q(x)
$$

and similarly for $q^{\prime}$ in $P^{\prime}$.

Let $u, u^{\prime} \in H^{2}(D)$ solve

$$
(\Delta+q) u=0, \quad\left(\Delta+q^{\prime}\right) u^{\prime}=0
$$

and $u=u^{\prime}$ outside $P \cup P^{\prime}$. Assume that $u\left(x_{c}\right) \neq 0$ or $u^{\prime}\left(x_{c}\right) \neq 0$ at every vertex $x_{c}$ of $P \cup P^{\prime}$. Then $P=P^{\prime}$ and

$$
\lim _{\substack{x \in P \\ x \rightarrow x_{c}}} q(x)=\lim _{\substack{x \in P \\ x \rightarrow x_{c}}} q^{\prime}(x) .
$$

Proof. If $P=\emptyset=P^{\prime}$ we are done. Otherwise use Lemma 3.6 with $\Omega=P \cup P^{\prime}$, $q, q^{\prime}, u, u^{\prime}$ all restricted to $\Omega$, and $x_{c}$ a vertex of $P$ (for example) that does not belong to $\overline{P^{\prime}}$. Since $u=u^{\prime}$ in $D \backslash \bar{\Omega}$ we have

$$
\left(q-q^{\prime}\right)\left(x_{c}\right) u\left(x_{c}\right)=\left(q-q^{\prime}\right)\left(x_{c}\right) u^{\prime}\left(x_{c}\right)=0
$$

where $q\left(x_{c}\right)$ and $q^{\prime}\left(x_{c}\right)$ are the limits of $q(x), q^{\prime}(x)$ as $x \rightarrow x_{c}$ in $\Omega$, and hence in $P$. Since $u\left(x_{c}\right) \neq 0$ or $u^{\prime}\left(x_{c}\right) \neq 0$ we get $q\left(x_{c}\right)=q^{\prime}\left(x_{c}\right)$. However $q^{\prime}$ is continuous in a neighbourhood of $x_{c}$ in $D$ because this point is away from $P^{\prime}$. Moreover $q^{\prime}=q$ outside $\Omega$. Thus

$$
\lim _{\substack{x \in P \\ x \rightarrow x_{c}}} q(x)=q\left(x_{c}\right)=q^{\prime}\left(x_{c}\right)=\lim _{\substack{x \in D \\ x \rightarrow x_{c}}} q^{\prime}(x)=\lim _{\substack{x \in D \backslash \bar{P} \\ x \rightarrow x_{c}}} q^{\prime}(x)=\lim _{\substack{x \in D \backslash \bar{P} \\ x \rightarrow x_{c}}} q(x)
$$

which is a contradiction.

Hence all vertices of $P$ belong to $\overline{P^{\prime}}$. Similarly we see that all vertices of $P^{\prime}$ belong to $\bar{P}$. Admissible cells are the interior of the convex hull of their vertices, so we have $P \subset P^{\prime} \subset P$ i.e. $P=P^{\prime}$. However we may then use Lemma 3.6 directly to see that $q\left(x_{c}\right)=q^{\prime}\left(x_{c}\right)$ because $q^{\prime}$ is not continuous anymore around $x_{c}$ in $D$.

For the recovery of the piecewise constant potentials we will also need a tool to propagate the equality of the total fields $u=u^{\prime}$ into cells where we have shown that the potentials are equal. This tool is Holmgren's uniqueness theorem.

Lemma 3.8. Let $U \subset \mathbb{R}^{n}$ be a connected Lipschitz domain. Let $\Gamma \subset \partial U$ be a non-empty relatively open subset of the boundary of $U$. Let $w \in H^{2}(U)$ satisfy $(\Delta+c) w=0$ in $U$ for some constant $c \in \mathbb{C}$ and assume that the Dirichlet and Neumann data of $w$ vanishes on $\Gamma$. Then $w=0$ in $U$.

Proof. Let $B \subset \mathbb{R}^{n}$ be a smooth open neighbourhood of a boundary point in $\Gamma$ such that $B \cap \partial U \subset \Gamma$. Let $w^{*}$ be the extension of $w$ by zero to $B$. It is a distribution. We will show that $(\Delta+c) w^{*}=0$ in $B$. Let $\varphi \in C_{0}^{\infty}(B)$. Then 
by noting that $(\Delta+c) w=0$ in $U$, and by the vanishing of the Cauchy data of $w$ on $\Gamma$, and the vanishing of the Cauchy data of $\varphi$ on $\partial B$, there holds

$$
\begin{aligned}
\int_{B} w^{*}(x)(\Delta+c) \varphi(x) d x=\int_{B \cap U} w(x)(\Delta+c) \varphi(x) d x \\
=\int_{\partial(B \cap U)}\left(w(x) \partial_{\nu} \varphi(x)-\varphi(x) \partial_{\nu} w(x)\right) d \sigma(x)=0 .
\end{aligned}
$$

Hence we have $(\Delta+c) w^{*}=0$ in $B$,

$$
B=(B \cap U) \cup(B \cap \Gamma) \cup(B \backslash \bar{U}),
$$

and $w^{*}=0$ in $B \backslash \bar{U}$ which is non-empty since Lipschitz domains are by definition on one side of their boundary. Holmgren's uniqueness theorem for distributions shows that $w^{*}=0$ in a neighbourhood of $B \cap \Gamma[16,[26]$, so $w=0$ in a non-empty open subset of $U$. It is real-analytic in $U$, and so must vanish everywhere there by connectedness.

Proof of Theorem 2.5. By the Rellich's theorem and unique continuation arguments of any of $[7,13,15,22]$ we have $u=u^{\prime}$ outside $P \cup P^{\prime}$, and so Proposition 3.7 implies $P=P^{\prime}$. Inside $P$ the total waves $u, u^{\prime}$ satisfy

$$
\left(\Delta+k^{2}(1+\varphi)\right) u=0, \quad\left(\Delta+k^{2}\left(1+\varphi^{\prime}\right)\right) u^{\prime}=0,
$$

because $\chi_{P}=1$ there. Lemma 3.6 shows then that

$$
k^{2}\left(\varphi-\varphi^{\prime}\right)\left(x_{c}\right) u\left(x_{c}\right)=k^{2}\left(\varphi-\varphi^{\prime}\right)\left(x_{c}\right) u^{\prime}\left(x_{c}\right)=0
$$

for any vertex $x_{c}$ of $P$. Since $u\left(x_{c}\right) \neq 0$ or $u^{\prime}\left(x_{c}\right) \neq 0$ we have $\varphi\left(x_{c}\right)=$ $\varphi^{\prime}\left(x_{c}\right)$.

Proof of Theorem 2.6. Write the potentials explicitely as

$$
V(x)=\sum_{j=1}^{\infty} V_{j} \chi_{\Sigma_{j}}, \quad V^{\prime}(x)=\sum_{j=1}^{\infty} V_{j}^{\prime} \chi_{\Sigma_{j}}
$$

for some sequences of constants $V_{j}, V_{j}^{\prime} \in \mathbb{C}$.

By Rellich's theorem and unique continuation the total waves $u, u^{\prime}$ satisfying

$$
\left(\Delta+k^{2}(1+V)\right) u=0, \quad\left(\Delta+k^{2}\left(1+V^{\prime}\right)\right) u^{\prime}=0
$$

are equal in $\operatorname{int}\left(\mathbb{R}^{n} \backslash \cup_{j} \overline{\Sigma_{j}}\right)$ since $\cup_{j} \overline{\Sigma_{j}}$ is simply connected. We shall denote this exterior domain by $\Sigma_{0}$. We will prove the following claim by induction: $V_{j}=V_{j}^{\prime}$ and $u=u^{\prime}$ in $\Sigma_{j}$ for $j<m$. The case $m=1$ is true with the interpretation that $V_{0}=V_{0}^{\prime}=0$.

Assume that the induction hypothesis holds for $j<m$. If $\Sigma_{m}=\emptyset$ then $V_{m}=V_{m}^{\prime}=0$ and the induction step is trivial. Otherwise let $x_{c}$ be a vertex of $\Sigma_{m}$ that's connected to infinity by a path that stays a uniform positive distance away from $\Sigma_{k}$ for $k>m$. This means that there is $r>0$ such that $B\left(x_{c}, r\right) \backslash \overline{\Sigma_{m}}$ belongs to $\Sigma_{0} \cup \ldots \cup \Sigma_{m-1}$. The induction assumption implies that $u=u^{\prime}$ on $B\left(x_{c}, r\right) \backslash \overline{\Sigma_{m}}$. Hence also $u=u^{\prime}$ and $\partial_{\nu} u=\partial_{\nu} u^{\prime}$ on $B\left(x_{c}, r\right) \cap \partial \Sigma_{m}$. Proposition 3.6 and $u\left(x_{c}\right) \neq 0$ or $u^{\prime}\left(x_{c}\right) \neq 0$ imply that $V_{m}=V_{m}^{\prime}$. Finally, Holmgren's uniqueness theorem of Lemma 3.8 implies that $u=u^{\prime}$ on $\Sigma_{m}$. The induction is complete. 
Proof of Theorem 2.7. Write out the nested structures of $V$ and $V^{\prime}$ as

$$
V=\sum_{j=1}^{\infty} V_{j} \chi_{\Sigma_{j}}, \quad V^{\prime}=\sum_{j=1}^{\infty} V_{j}^{\prime} \chi_{\Sigma_{j}^{\prime}}
$$

where $\Sigma_{j}=D_{j} \backslash \overline{D_{j+1}}$ for admissible cells or empty sets $D_{j}$ with

$$
D_{j} \ni D_{j+1} \text {. }
$$

Similarly for $V^{\prime}$.

Let us prove that $D_{j}=D_{j}^{\prime}, V_{j}=V_{j}^{\prime}$ when $j<m$ and $u=u^{\prime}$ outside $D_{m} \cup D_{m}^{\prime}$ by induction on $m$. Interpret $D_{0}=D_{0}^{\prime}=\mathbb{R}^{n}, V_{0}=V_{0}^{\prime}=0$. By the Rellich's theorem and unique continuation arguments of any of $7,13,15,22$ we have $u=u^{\prime}$ outside $D_{1} \cup D_{1}^{\prime}$ and so the case $m=1$ is true.

Let the above claim hold for some fixed $m$. We have $u=u^{\prime}$ outside $D_{m} \cup D_{m}^{\prime}$. Moreover $V_{m} \neq V_{m-1}=V_{m-1}^{\prime} \neq V_{m}^{\prime}$ so $V$ and $V^{\prime}$ have jumps at the vertices of $D_{m}$ and $D_{m}^{\prime}$, respectively and are equal outside $D_{m} \cup D_{m}^{\prime}$. Proposition 3.7 implies $D_{m}=D_{m}^{\prime}$ and $V_{m}=V_{m}^{\prime}$. The same conclusion holds also when either of them is the empty set after which we may stop. By Holmgren's uniqueness theorem (Lemma 3.8) we have $u=u^{\prime}$ in $D_{m}$ । $\left(D_{m+1} \cup D_{m+1}^{\prime}\right)$ and so anywhere outside $D_{m+1} \cup D_{m+1}^{\prime}$. The induction step is complete.

\section{ACKNOWLEDGEMENT}

The work of H Liu was supported by a startup fund from City University of Hong Kong and the Hong Kong RGC general research funds (projects $12302017,12301218,12302919)$. The authors would like to thank the anonymous referee for the constructive comments and suggestions, which have lead to significant improvements on the presentation and the result of the paper.

\section{REFERENCES}

[1] Akopyan, A., BÁrÁny, I. and Robins, S.: Algebraic vertices of non-convex polyhedra, Advances in Mathematics, 308 (2017), 627-644.

[2] Blåsten, E., Tzou, L. and WAng, J. N.: Uniqueness for the inverse boundary value problem with singular potentials in 2D, arXiv:1704.06397

[3] E. Blåsten, X. Li, H. Liu and Y. WAnG, On vanishing and localization near cusps of transmission eigenfunctions: a numerical study, Inverse Problems, 33 (2017), 105001.

[4] BlÅsten, E. and Liu, H.: On vanishing near corners of transmission eigenfunctions, J. Funct. Anal., 273 (2017), 3616-3632. Addendum, arXiv:1710.08089.

[5] Blåsten, E. and LiU, H.: On corners scattering stably and stable shape determination by a single far-field pattern, Indiana Univ. Math. J, in press, 2019.

[6] Blåsten, E., Imanuvilov, O. Y. and Yamamoto, M.: Stability and uniqueness for a two-dimensional inverse boundary value problem for less regular potentials, Inverse Problems and Imaging, 9 (2015), 709-723.

[7] Blåsten, E., Päıvärinta, L., and Sylvester, J.: Corners always scatter, Comm. Math. Phys., 331 (2014), 725-753.

[8] Bukhgeim, A. L.: Recovering a potential from Cauchy data in the two-dimensional case, J. Inverse Ill-posed Probl., 16 (2008), 19-33.

[9] Colton, D. and Kress, R.: Inverse Acoustic and Electromagnetic Scattering Theory, 2nd Ed., Springer, New York, 1998. 
[10] Colton, D. and Monk, P.: The inverse scattering problem for time-harmonic acoustic waves in an inhomogeneous medium, The Quarterly Journal of Mechanics and Applied Mathematics, Vol 41 (1988), 97-125.

[11] Colton, D., PÄIvÄrinta, L. and Sylvester, J.: The interior transmission problem, Inverse Probl. Imaging, Vol 1 (2007), 13-28.

[12] Dos Santos Ferreira, D., Kenig, C. E. and Salo, M.: Determining an unbounded potential from cauchy data in admissible geometries, Comm. PDE, 38 (2013), 50-68.

[13] Elschner, J. and Hu, G.: Corners and edges always scatter, Inverse Problems, 31 (2015), 015003, 1-17.

[14] Hu, G., LI, J., and LiU, H.: Uniqueness in determining refractive indices by formally determined far-field data, Appl. Anal., 94 (2015), 1259-1269.

[15] Hu, G., Salo, M., and Vesalainen, E.: Shape Identification in Inverse Medium Scattering, SIAM J. Math. Anal., 48 (2016), 152-165.

[16] Holmgren, E.: Über Systeme von linearen partiellen Differentialgleichungen, Öfversigt af Kongl. Vetenskaps-Academien Förhandlinger, 58 (1901), 91-103.

[17] Isakov, V.: Inverse Problems for Partial Differential Equations, 2nd Ed., SpringerVerlag, New York, 2006.

[18] Jerison, D. and Kenig, C. E.: Unique continuation and absence of positive eigenvalues for Schrödinger operators, Annals of Mathematics, 121 (1985), 463-488.

[19] Kusiak, S. and Sylvester, J.: The Scattering Support, Comm. Pure Appl. Math, 56 (2003), 1525-1548.

[20] LiU, H. and LiU, X.: Recovery of an embedded obstacle and its surrounding medium by formally-determined scattering data, Inverse Problems, 33 (2017), 065001.

[21] NaChman, A.: Reconstructions from boundary measurements, Ann. of Math. (2), 128 (1988), 531-576.

[22] Pärvärinta, L., Salo, M. and Vesalainen, E.: Strictly convex corners scatter, Rev. Mat. Iberoamericana, in press.

[23] RAKesh and Uhlmann, G.: Uniqueness for the inverse back-scattering problem for angularly controlled potentials, Inverse Problems, 30 (2014), 065005.

[24] RAKesh and Uhlmann, G.: The point source inverse back-scattering problem, Contemporary Mathematics, 644 (2015).

[25] Sylvester, J. and Uhlmann, G.: A global uniqueness theorem for an inverse boundary value problem, Ann. of Math. (2), 125 (1987), 153-169.

[26] Treves, F.: Introduction to pseudodifferential and Fourier integral operators, Vol. 1, Plenum Press, New York, 1980.

[27] Uhlmann, G.: 30 years of Calderón's problem, Séminaire Laurent SchwartzÉquations aux dérivées partielles et applications, Année 2012-2013, Exp. No. XIII, 25 pp., Sémin. Équ. Dériv. Partielles, École Polytech., Palaiseau, 2014.

[28] Uhlmann, G.: Inverse boundary value problems and applications, Méthodes semiclassiques, Vol. 1, Astérisque, 2017 (1992), 153-211.

Department of Mathematics, University of Helsinki, Helsinki, Finland E-mail address: emilia.blasten@iki.fi

Department of Mathematics, City University of Hong Kong, Kowloon, Hong Kong SAR, CHINA.

E-mail address: hongyu.liuip@gmail.com; hongyliu@cityu.edu.hk 